D.O.I.: $10.3895 / \mathrm{S} 1808-04482008000100004$

\title{
CUSTOS E PROJETOS DE SISTEMAS NA INDÚSTRIA DA CONSTRUÇÃO CIVIL
}

\section{COSTS AND PROJECTS OF SYSTEMS IN THE INDUSTRY OF BUILDING}

\author{
Márcio Botelho da Fonseca Lima ${ }^{1}$; Luiz Bueno da Silva ${ }^{2}$; Glauco S. R. Gomes ${ }^{3}$ \\ ${ }^{1}$ Federal University da Paraíba-UFPB -João Pessoa - Brasil tismalu@uol.com.br \\ ${ }^{2}$ Federal University da Paraíba-UFPB -João Pessoa - Brasil bueno@ct.ufpb.br \\ ${ }^{3}$ Federal University da Paraíba-UFPB -João Pessoa - Brasil gomesjpa@terra.com.br
}

\section{Resumo}

Este artigo procura adicionar contribuições da Economia Industrial às técnicas utilizadas no domínio da Gestão de Projetos. Primeiro, apresenta-se a técnica da força bruta direcionada à construção civil e aprimorada pela utilização do MSProject. Segundo, são desenvolvidos modelos de minimização do tempo de finalização da compressão do projeto e de programação da compressão que poderia implicar o mínimo custo adicional. Terceiro, são estabelecidas leis de custos que permitem inferir que o custo de um projeto não depende apenas da taxa de produção. Concluindo, é salientada a importância da Gestão de Projeto com respeito à procura de soluções dinâmicas e inovadoras aos problemas produtivos mais importantes desta era do conhecimento.

Palavras-chave: Compressão de projetos, construção civil, sistemas irregulares de produção.

\section{Introdução}

A compressão de um projeto diz respeito a uma avaliação dos custos de redução da duração das atividades que estão no caminho crítico. Após a realização dessa avaliação, as atividades que tiverem o menor custo para a redução de sua duração deverão ser trabalhadas. Isso significa dizer que o aporte de mais recursos financeiros, humanos (horas extras, por exemplo), materiais ou equipamentos geralmente acarretará um aumento do orçamento do projeto (MENEZES, 2003).

A segunda seção deste artigo apresenta o método da força bruta, utilizado para efetuar o crashing (compressão ou aceleração) de um "projeto da casa", exemplo extraído da literatura de gestão de projetos, especificamente de Ragsdale (2001).

É importante enfatizar que o MSProject acelera consideravelmente o desenvolvimento desse método, bem como aplicações de inúmeros outros métodos de planejamento, programação e controle de projetos (VARGAS, 2004; WIRTH, 2001; FIGUEIREDO \& FIGUEIREDO,1999). Ressalte-se que a técnica supracitada é completamente atendida pelo MSProject.

A terceira seção apresenta algumas aproximações de programação linear com a intenção de 
determinar o menor tempo possível para a conclusão de um projeto e para programar a compressão do projeto que implicaria no menor custo adicional, dada uma restrição imposta por condições de contrato, por exemplo, um tempo de conclusão pré-determinado.

Algumas discussões acerca de trade-offs relativos a custos de compressão e duração de projetos também são tratadas. Segundo Sandroni (1999), em economia, trade-off significa expressão que define situação de escolha conflitante, isto é, quando uma ação econômica que visa à resolução de determinado problema acarreta, inevitavelmente, outros.

Na quarta seção, já no âmbito da Economia Industrial e da Inovação, Gaffard (1990) destaca custos e produtos numa ótica temporal, mediante o estabelecimento das chamadas leis de custos que aprofundam reflexões concernentes à compressão de projetos.

Enfim constata-se que, na era do conhecimento, o papel a ser desempenhado pela Gestão de Projetos nesse processo de tomada de decisão será cada vez mais relevante.

\section{O método da força bruta}

Este é o método mais conhecido, utilizado para a compressão de um projeto, discutido por vários autores, dentre eles, Cassaroto Filho et al (19922). O método é simples, lento e passível de falhas, o que o torna inadequado para grandes projetos. Os passos para o método da força bruta são:

a) Seleciona-se a atividade com menor custo diário de compressão;

b) A atividade é reduzida de uma unidade de tempo;

c) Utiliza-se a tabela 2 para verificar o custo diário de compressão relacionado à atividade selecionada;

d) Os tempos de início e término da rede de atividades são recalculados. Também é verificado se a redução de uma unidade de tempo na duração da atividade acelerada não modifica o caminho crítico;

e) As atividades b, c, e d são repetidas até que a duração da máxima aceleração seja obtida, ou até que um novo caminho crítico surja na rede de atividades;

f) No caso do surgimento de um novo caminho crítico na rede de atividades, deve haver dois caminhos críticos paralelos. Logo, para a redução do tempo do projeto em mais uma unidade de tempo, é necessário a compressão simultânea das atividades críticas com o menor custo diário de compressão em cada um dos caminhos críticos. Assim sendo, duas parcelas estão sendo somadas aos custos do projeto. É necessário verificar se a soma dos custos diários é menor que o custo marginal de uma outra atividade ainda não acelerada.

As tabelas 1 e 2 resumem os dados relativos ao projeto de construção da casa, exemplo extraído de Ragsdale (2001). 


\begin{tabular}{|c|c|c|c|c|c|}
\hline $\begin{array}{l}\text { ATIVI- } \\
\text { DADE }\end{array}$ & DES CRIÇÄO & DURAÇÄO & INICIO & TÉRMINO & $\begin{array}{c}\text { PREDEC ESSO- } \\
\text { RAS }\end{array}$ \\
\hline & Construção de Casa & $46 d$ & Ter $7 / 12 / 04$ & $\operatorname{Sex} 21 / 1 / 05$ & \\
\hline & Início & Dd & Ter $7 / 12 / 04$ & & \\
\hline A & Escavação & $3 \mathrm{~d}$ & Ter $7 / 12 / 04$ & $\operatorname{Sex} 21 / 1 / 05$ & 2 \\
\hline $\mathrm{B}$ & Fundação & $4 d$ & Sex 10/12/04 & & 3 \\
\hline $\mathrm{C}$ & Encanamento preliminar & $3 \mathrm{~d}$ & Ter $14 / 12 / 04$ & $\operatorname{Sex} 21 / 1 / 05$ & 4 \\
\hline$\overline{\mathrm{D}}$ & Estrutura & $10 \mathrm{~d}$ & Ter $14 / 12 / 04$ & & 4 \\
\hline$E$ & Acabamento exterior & $8 \mathrm{~d}$ & Sex 24/12/04 & $\operatorname{Sex} 21 / 1 / 05$ & 6 \\
\hline $\mathrm{F}$ & Instal. hidro-sanitária & $4 d$ & Sex 24/12/04 & & $\frac{8}{6}$ \\
\hline $\mathrm{G}$ & Instal. elétrica preliminar & $6 \mathrm{~d}$ & $\operatorname{Sex} 24 / 12 / 04$ & $\operatorname{Sex} 21 / 1 / 05$ & 6 \\
\hline $\mathrm{H}$ & Revestimento & $8 d$ & Sáb 1/1/05 & & $15 ; 7 ; 8 ; 9 "$ \\
\hline I & Carpintaria & $5 \mathrm{~d}$ & Dom 9/1/05 & $\operatorname{Sex} 21 / 1 / 05$ & 10 \\
\hline $\mathrm{J}$ & Pintura & $5 \mathrm{~d}$ & Dom 9/1/05 & & 10 \\
\hline $\mathrm{K}$ & Encanamento final & $4 d$ & Sex 14/1/05 & $\operatorname{Sex} 21 / 1 / 05$ & 11 \\
\hline $\mathrm{L}$ & Instal. Elétrica final & $2 \mathrm{~d}$ & Sex 14/1/05 & & 12 \\
\hline $\mathrm{MI}$ & Pisos & $4 d$ & Ter $18 / 1 / 05$ & $\operatorname{Sex} 21 / 1 / 05$ & "13;14" \\
\hline $\mathrm{N}$ & Fim & 0d & $\operatorname{Sex} 21 / 1 / 05$ & & $" 14 ; 15 "$ \\
\hline
\end{tabular}

A tabela 1 ilustra o modo de exibição "Gráfico de Gantt" do MS Project, onde foram digitadas as descrições das atividades, suas respectivas durações e suas atividades predecessoras.

\begin{tabular}{|c|c|c|c|c|c|c|}
\hline \multirow[b]{2}{*}{ ATIVIDADE } & \multirow[b]{2}{*}{ DESCRIÇÃO } & \multicolumn{2}{|c|}{ NORMAL } & \multicolumn{2}{|c|}{ ACELERADO } & \multirow{2}{*}{$\begin{array}{c}\text { CUSTO DIÁRIO } \\
\text { DE } \\
\text { COMPRESSÃO }\end{array}$} \\
\hline & & $\begin{array}{c}\text { Duração } \\
\text { (Dias) }\end{array}$ & $\begin{array}{l}\text { Custo } \\
\text { (R\$) }\end{array}$ & $\begin{array}{r}\text { Duração } \\
\text { (Dias) }\end{array}$ & $\begin{array}{l}\text { Custo } \\
\text { (R\$) }\end{array}$ & \\
\hline $\mathrm{A}$ & Escavação & 3 & 5.000 & 2 & 6.000 & 1.000 \\
\hline $\mathrm{B}$ & Fundação & 4 & 12.000 & 3 & 15.000 & 3.000 \\
\hline $\mathrm{C}$ & Encanamento preliminar & 3 & 3.000 & 2 & 3.500 & 500 \\
\hline $\mathrm{D}$ & Estrutura & 10 & 20.000 & 6 & 25.000 & 1.250 \\
\hline$E$ & Acabamento exterior & 8 & 8.000 & 5 & 10.000 & 667 \\
\hline $\mathrm{F}$ & Instalação hidro-sanitários. & 4 & 11.000 & $\frac{6}{3}$ & 12.000 & 1.000 \\
\hline $\mathrm{G}$ & Instal. elétrica preliminar & 6 & 3.500 & 4 & 4.500 & 500 \\
\hline $\mathrm{H}$ & Revestimento & 8 & 5.000 & 5 & 6.500 & 500 \\
\hline I & Carpintaria & 5 & 8.000 & 3 & 9.500 & 750 \\
\hline $\mathrm{J}$ & Pintura & 5 & 4.000 & 2 & 5.500 & 500 \\
\hline $\mathrm{K}$ & Encanamento final & 4 & 7.000 & 2 & 8.500 & 750 \\
\hline $\mathrm{L}$ & Instalação Elétrica final & 2 & 2.000 & 1 & 2.500 & 500 \\
\hline $\mathrm{M}$ & Pisos & 4 & 10.000 & 2 & 12.000 & 1.000 \\
\hline $\mathrm{N}$ & Totais & 46 & 98.500 & 28 & 116.834 & \\
\hline
\end{tabular}

Tabela 2: Durações e custos normal e acelerado do projeto da casa

A tabela 2 fornece os dados iniciais do projeto da casa referentes às descrições das atividades, às suas respectivas durações normais e aceleradas bem como os seus respectivos custos. Além disso, facilmente, são calculados os custos diários de compressão de cada atividade.

A tabela 3 e a figura 1 resumem os principais procedimentos realizados no exemplo da compressão do projeto da casa, desenvolvido pelo método da força bruta, com o auxílio do MSProject. 


\begin{tabular}{|c|c|c|c|}
\hline Projetos & Compressão (dias) & Tempo de finalização (dias) & Custo adicional (R\$) \\
\hline Proj. da casa & Duração normal & 46 & 0 \\
\hline Proj. da casa 1 & Atividade $\mathrm{H}-1$ dia & 45 & 500 \\
\hline Proj. da casa 2 & Atividade $\mathrm{H}-1$ dia & 44 & 1.000 \\
\hline Proj. da casa 3 & Atividade $\mathrm{H}-1$ dia & 43 & 1.500 \\
\hline Proj. da casa 4 & Atividade $\mathrm{H}-1$ dia & 42 & 2.167 \\
\hline Proj. da casa 5 & Atividade E-1 dia & 41 & 2.834 \\
\hline Proj da casa 6 & Atividade E-1 dia & 40 & 3.584 \\
\hline Proj. da casa 7 & Atividade G-1 dia & 39 & 4.084 \\
\hline Proj. da casa 8 & Atividade $\mathrm{K}-1$ dia & 38 & 4.834 \\
\hline Proj. da casa 9 & $\begin{array}{l}\text { Atividade } \mathrm{I}_{\text {- }}-1 \text { dia } \\
\text { Atividade } \mathrm{J}_{-1}-1 \text { dia }\end{array}$ & 37 & 6.084 \\
\hline Proj. da casa 10 & $\begin{array}{l}\text { Atividade } \mathrm{I}_{\mathrm{w}}-1 \text { dia } \\
\text { Atividade } \mathrm{J}_{-1}-1 \text { dia }\end{array}$ & 36 & 7.334 \\
\hline Proj. da casa 11 & Atividade $A-1$ dia & 35 & 8.334 \\
\hline Proj. da casa 12 & Atividade M-1 dia & 34 & 9.334 \\
\hline Proj da casa 13 & Atividade $M-1$ dia & 33 & 10.334 \\
\hline Proj. da casa 14 & Atividade D-1 dia & 32 & 11.584 \\
\hline Proj. da casa 15 & Atividade D-1 dia & 31 & 12.834 \\
\hline Proj. da casa 16 & Atividade D-1 dia & 30 & 14.084 \\
\hline Proj. da casa 17 & Atividade D-1 dia & 29 & 15.334 \\
\hline Proj. da casa 18 & Atividade B-1 dia & 28 & 18.334 \\
\hline
\end{tabular}

Tabela 3: Procedimentos efetuados no MSProiect para o projeto da casa,

A tabela 3 resume passo a passo os procedimentos utilizados para calcular-se o tempo de finalização do projeto em dias e o custo adicional total $(\mathrm{R} \$ 18.334,00)$.

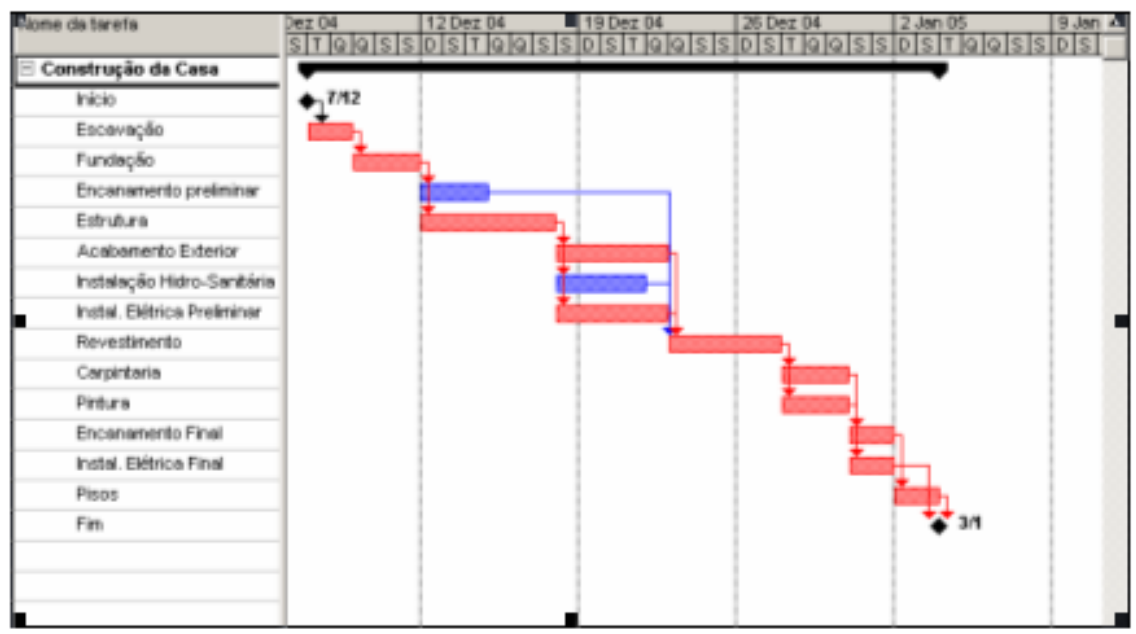

Figura 1: Gráfico de Gantt da compressão do projeto para 28 dias, a partir do método da força bruta (projeto da casa 18).

Finalizando, o método da força bruta é simples, porém manual, demorado e sujeito a erros freqüentes, caso não se utilizem ferramentas como o MS Project. Desta maneira, a duração do projeto foi reduzida de 46 dias para 28 dias, acarretando um custo adicional total de compressão igual a $\mathrm{R} \$ 18.334,00$.

\section{Modelos de programação linear relativos à compressão de projetos}




\subsection{Modelo de determinação do menor prazo possível para a conclusão do projeto}

De acordo com Ragsdale (2001), o gerente de projetos desejaria saber a data mais cedo possível necessária de aceleração de modo a satisfazer a data final do projeto. A formulação de um modelo de programação linear destinado a solucionar este problema é disposta na figura 2.

A função objetivo (1) tenta minimizar o total de tempo necessário para a conclusão do projeto. As restrições (2) - (17) garantem que certa atividade só tenha início quando sua atividade predecessora tenha sido realizada. A restrição (18) garante que o tempo de compressão de uma atividade não viole o limite estabelecido. Finalmente, a restrição (19) está relacionada à natureza das variáveis.

Observa-se na implantação do modelo que a duração do projeto de 28 dias corresponde a um custo de compressão de $\$ 19.000,00$, levemente diferente daquele obtido com o método da força bruta $(\$ 18.334,00)$.

\section{2}

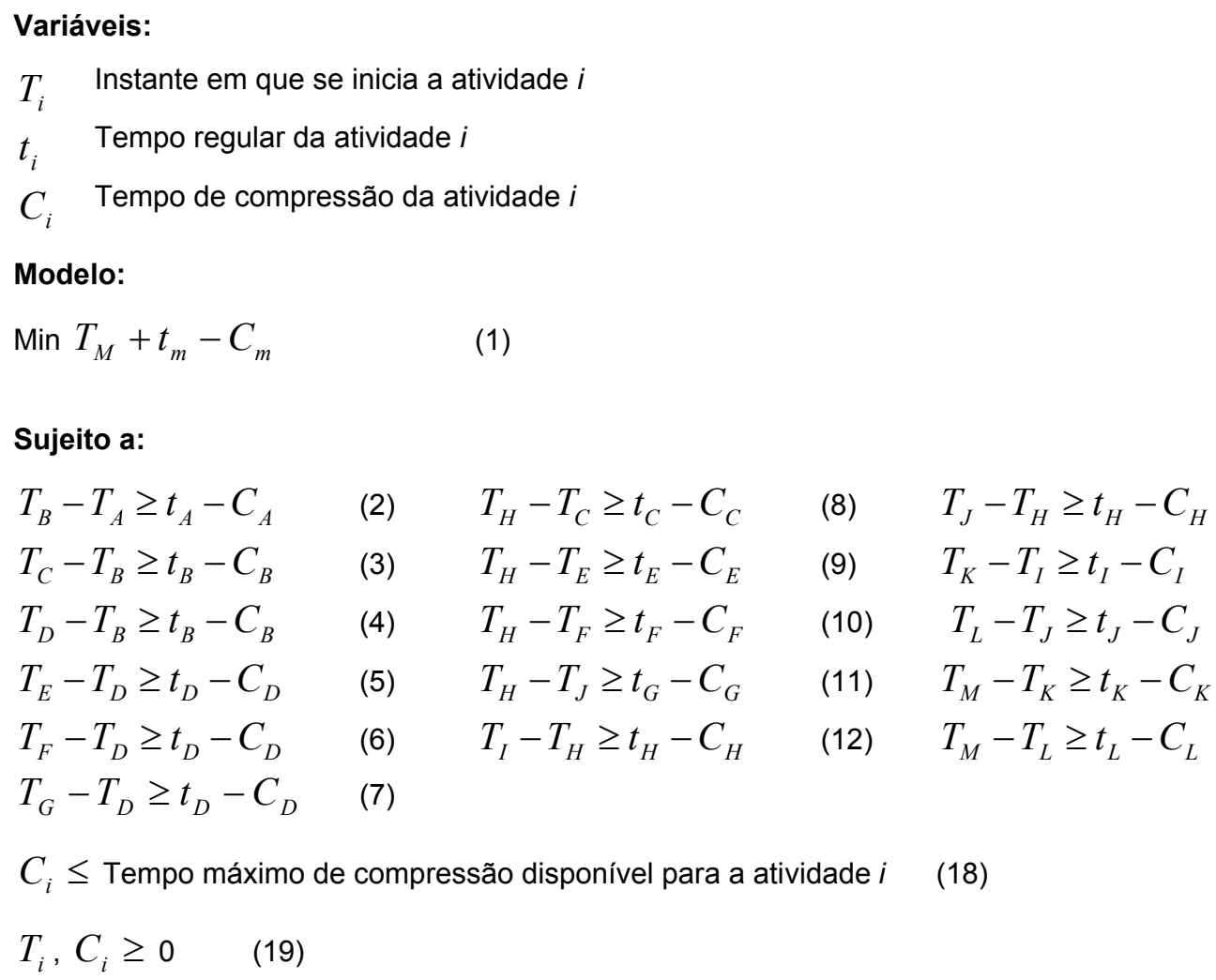

Sujeito a:

$T_{B}-T_{A} \geq t_{A}-C_{A}$

(2) $T_{H}-T_{C} \geq t_{C}-C_{C}$

(8) $T_{J}-T_{H} \geq t_{H}-C_{H}$

$T_{C}-T_{B} \geq t_{B}-C_{B}$

$T_{H}-T_{E} \geq t_{E}-C_{E}$

(9) $\quad T_{K}-T_{I} \geq t_{I}-C_{I}$

$T_{D}-T_{B} \geq t_{B}-C_{B}$

(4) $T_{H}-T_{F} \geq t_{F}-C_{F}$

(10) $\quad T_{L}-T_{J} \geq t_{J}-C_{J}$

$T_{E}-T_{D} \geq t_{D}-C_{D}$

$T_{H}-T_{J} \geq t_{G}-C_{G}$

$T_{M}-T_{K} \geq t_{K}-C_{K}$

$T_{F}-T_{D} \geq t_{D}-C_{D}$

$T_{I}-T_{H} \geq t_{H}-C_{H}$

$T_{M}-T_{L} \geq t_{L}-C_{L}$

$T_{G}-T_{D} \geq t_{D}-C_{D}$

$C_{i} \leq$ Tempo máximo de compressão disponível para a atividade $i$

$T_{i}, C_{i} \geq 0$

Figura 2 - Modelo de programação linear

\section{Incluindo um limite de tempo para a conclusão do projeto}

É importante salientar que, em certas situações, o tempo especificado em contrato (Tp), pode ser menor do que o tempo normal de execução do projeto (Tn); em outras palavras, sem 
compressão. Além disso, também é considerada uma multa para cada dia transpassado à data estabelecida em contrato. Tais características conduzem o modelo a uma realidade mais próxima da gestão de projetos.

Entretanto surgem problemas conflitantes acerca do custo total do projeto e do tempo de compressão das atividades. Por exemplo, pode ocorrer um trade-off entre os elementos citados. Logo, a seguinte questão deve ser respondida: é vantajoso comprimir suficientemente o projeto, de modo a alcançar Tp, ou comprimir (ou não) certa quantidade de tempo e pagar a multa?

Verifica-se que o modelo apresentado previamente não atende às condições mencionadas acima. Assim, a função objetivo (1) foi modificada de maneira a ajustar-se às características relativas ao tempo de projeto especificado em contrato e à existência da multa $\mathrm{P}$.

Então:

$$
\operatorname{Min} \sum_{i=A}^{M} C C_{i} \cdot C_{i}-P\left(T_{e}-T_{p}\right)
$$

Onde $C C_{i}$ é o custo de compressão da atividade $i$, e $T_{e}$ é o tempo de execução do projeto (onde $T_{e} \geq T_{p}$ ).

A nova função objetivo (20) tenta minimizar a soma dos custos de compressão e ao mesmo tempo adiciona uma multa diária constante se $T_{e} \geq T_{p}$. Em outras palavras, sempre que $T_{e}$ aumenta em uma unidade de tempo, a função objetivo aumenta no valor de $\mathrm{P}$.

O modelo foi implementado utilizando-se o LINDO 6.1 e os seguintes resultados foram obtidos:

- $\quad$ Tempo de projeto $=43$ dias;

- $\quad$ Custo de compressão $=\$ 1.500,00$;

$-\quad$ Multa $=\$ 1.500,00$.

Existem algumas situações onde surge a necessidade de se cumprir alguma atividade específica em certo horizonte de tempo determinado por contrato. Suponha que, no exemplo da construção da casa a atividade D deva ser realizada até o $16^{\circ}$ dia do projeto. Para tal, a seguinte restrição deve ser adicionada ao modelo:

$$
T_{d}+t_{d}-C_{d} \leq 16
$$

Os resultados obtidos após a inclusão da restrição (21), foram:

- Tempo de projeto $=42$ dias

- $\quad$ Custos de compressão $=\$ 2.500,00$;

$-\quad$ Multa $=1.000,00$. 


\subsection{Considerações}

Observa-se que cada um dos métodos apresentados neste artigo possui características distintas para resolver o mesmo problema. Não é objetivo deste trabalho discutir qual deles é mais eficiente, e sim o mais eficaz de acordo com a realidade do gerente de projetos.

Matta \& Ashkenas discutem a utilização de planos de projeto, cronogramas e orçamentos de modo a reduzir o "risco de execução". Este risco está relacionado à possibilidade de atividades planejadas não serem executadas apropriadamente.

Os autores observam ainda que muitos tomadores de decisão negligenciam dois outros erros críticos: "risco do espaço em branco" que está associado ao risco de que algumas atividades necessárias não sejam identificadas previamente e, com isso, resultem em lacunas no plano do projeto; e o "risco de integração" que diz respeito ao risco de que atividades muito diferentes não sejam completadas simultaneamente no fim do projeto.

Assim, observa-se o grande potencial de uso dos métodos apresentados aqui como instrumentos de suporte no gerenciamento de projetos e na minimização dos erros supracitados, cada um deles conservando suas peculiaridades.

A programação linear apresenta diversas vantagens. Permite, por exemplo, a construção de modelos flexíveis que levam em consideração características específicas de um dado projeto. Algumas das desvantagens incluem a necessidade de know-how para a construção dos modelos e conhecimento de técnicas de programação matemática assim como dos softwares utilizados para a implementação dos modelos.

\section{Custos e produtos numa ótica temporal: a nova acepção das noções de custo e produto}

Os custos e produtos, quando são apreendidos segundo sua dimensão temporal, têm uma acepção inteiramente diferente daquela que é habitualmente retida. Esta outra acepção tem como particularidade o fato de que ela insere a teoria do capital no coração da teoria da firma; ela permite definir leis ou proposições que são coerentes com a observação empírica que, na maioria das vezes, são concernentes aos sistemas irregulares (GAFFARD, 1990).

Nos sistemas regulares ou permanentes de produção a empresa industrial é, em princípio, tecnologicamente eficaz, de tal maneira que os custos suportados no período corrente são, analiticamente, imputáveis à produção corrente. Em suma, grosso modo, as receitas estão sincronizadas com as despesas e a dimensão temporal não acrescenta muita coisa à análise de custos. As empresas que operam nesse sistema consistem, na maioria das vezes, de indústrias, onde operações em cadeia proporcionam ciclos de fabricação de produtos cujos tempos de produção são denominados períodos elementares. 
No sistema irregular ou transitório de produção, ao contrário, a empresa não é mais tecnologicamente eficaz, pois ela geralmente se encontra numa situação de construção de uma nova capacidade produtiva, onde os fundos não são plenamente utilizados, devido, em alguns casos, ao problema de aprendizagem de uma nova tecnologia. Aqui, a noção de fundos (ao contrário de fluxos) corresponde aos elementos que participam do processo de produção sem serem destruídos, por exemplo, a terra, o capital físico (prédios, máquinas e equipamentos) e os recursos humanos. No caso do carvão, na medida em que ele é consumido, tratar-se-ia de um exemplo de fluxo.

Nos sistemas irregulares existe, então, uma defasagem entre o perfil dos custos e o perfil da produção, que faz com que, analiticamente, os custos correntes não poderiam ser imputados à produção corrente, fato que levaria a tomar-se em consideração a dimensão temporal de maneira inequívoca. Com efeito, os custos suportados inicialmente para construir uma nova capacidade produtiva somente serão recuperados mais tarde quando a produção de bens tornar-se-á efetiva: esse intervalo de tempo entre custos e produtos, que não tem real importância no sistema regular, tornase essencial nos sistemas irregulares.

No contexto de tal sistema irregular não existe uma função de produção bem definida, devido ao fato de que a tecnologia não é dada a priori e está em curso de constituição. $\mathrm{O}$ instrumento analítico adequado parece ser uma função de custos que, naturalmente, não é mais derivada da função de produção e então não mais exprime situações de eficácia tecnológica. Esta função de custos deve permitir ressaltar o papel do tempo, de tal maneira que ela esteja inscrita numa perspectiva analítica que é aquela dos modelos temporais de produção.

\subsection{Custo de produção e modificação do valor do capital}

A maneira segunda a qual a dimensão temporal pode ser introduzida consiste em definir os custos como a modificação do valor do capital social da firma, resultante de alguma operação particular, supondo que a transformação da receita é omitida no cálculo da variação do valor do capital social.

Um exemplo que permite ilustrar essa definição seria: supondo que o valor atual dos ativos de uma empresa seja \$100 no início de uma operação, e deveria ser \$80 um ano após, no final da operação, não levando em consideração a venda dos produtos desta operação no mercado; o valor atual desses $\$ 80$, onde, por exemplo, a uma taxa de atualização de $6 \%$, seria de $\$ 75,47$. O custo da operação em valor presente do capital é de $\$ 24,53(100$ - 75,47).

A expressão do custo em unidade do capital social está aqui inserida numa abordagem "fundista" da avaliação da empresa, oposta no caso presente a uma abordagem materialista . A expressão do custo em unidade do capital social é, com efeito, coerente com a idéia de que o capital 
não é assimilável a sua contrapartida em ativos imobilizados, mas constitui um valor monetário que é somente o valor dos benefícios futuros que os acionários podem esperar.

Grosso modo, a abordagem "fundista" do capital baseia-se no fato de que, sob um ponto de vista estratégico, a detenção de ativos fixos pelas empresas constitui para elas um engajamento irreversível, em relação aos benefícios futuros que os acionistas podem esperar. Isto é especialmente verdadeiro para os casos em que a detenção de máquinas torna-se imperiosa pela dificuldade de substituí-las em curto espaço de tempo por intermédio do mercado. Isto é igualmente verdadeiro nos casos em que os recursos humanos são considerados como recursos coletivos.

A abordagem materialista basicamente consiste no fato de que o capital não é assimilável à sua contrapartida em ativos imobilizados, a exemplo dos fundos de depreciação, que são reservas em dinheiro formadas pelas empresas e regulamentadas por lei. São supridas pela taxa de depreciação do ativo fixo da empresa (imóveis, máquinas e equipamentos) e destinam-se à renovação desse ativo.

A produção é, portanto, definida como uma operação que se propaga no tempo, isto é, como um programa cujas características são as seguintes:

- Uma taxa de produção $x(t)$ que é geralmente o único aspecto considerado na análise econômica standard;

- O volume total de produção $(V)$ acumulado durante o programa de produção;

- A duração da operação de produção $(m)$.

Essas três características são resumidas na fórmula seguinte:

$$
V=\sum_{T}^{T+m} x(t) d t
$$

Onde $V$ é o volume total de produção, $x(t)$ a taxa de produção no instante $t, T$ o momento em que a primeira unidade de produto é fornecida, e m o intervalo de tempo durante o qual a produção torna-se possível.

Cada uma das características mencionadas para designar a operação de produção pode afetar o custo de produção. No caso de projetos de construção de produto único, a exemplo da produção de um navio, $x(t)$ seria igual a 1 unidade/ano, por exemplo, enquanto $T$ e m seriam coincidentes no tempo, fato pelo as fórmulas abaixo não teriam muita serventia. Nos projetos de construções de diversas residências pequenas, como no caso adotado neste artigo, $T$ poderia ser 45 dias, $x(t)$ seriam 9 unidades/ano e m seria igual a 40 dias, por exemplo.

\subsection{Os custos de produção fora do sistema permanente: as leis de custos}

Sobre a base das definições precedentes, Alchian (1977) elabora um conjunto de 
proposições sobre as maneiras pelas quais os custos são afetados por uma variação dessas variáveis ou características. Naturalmente, dentre as variáveis $V, x(t), T$ e $m$ somente três são independentes, a quarta sendo então restrição contrariamente ao sistema permanente onde as quatro características são invariantes (o que justifica o fato de que só se retém apenas uma dentre elas na análise). Seja $C$ a função de custo (isto é, de modificação de valor do capital social) tal que, $C=F(V, x, T, m)$.

Proposição 1:

$$
\frac{\partial C}{\partial x(t)}>0 \quad T=T_{0} ; V=V_{0}
$$

Os custos aumentam na medida em que a taxa $x(t)$, segundo a qual um volume é produzido, é mais elevada, o período de fabricação do produto $\mathrm{m}$, sendo reduzido em conseqüência. No projeto da casa adotado neste artigo, permanecendo $T$ igual a 45 dias, e $V$ sendo fixado em 11 residências, $x(t)$ poderia passar de 9 unidades/ano para 11 unidades/ano, $m$ seria então reduzido de 40 dias para 32 dias, na medida em que a firma teve de suportar um custo mais elevado, devido ao pagamento de horas extras, por exemplo.

Proposição 2:

$$
\frac{\partial^{2} C}{\partial x^{2}}>0 \quad T=T_{0} ; V=V_{0}
$$

O crescimento dos custos é uma função crescente da taxa de produção. Isto é, se o custo adicional incorrido para passar de $x(t)=9$ casas/ ano para $x(t)=11$ casas/ano for de $\$ 8.000,00$, a passagem de $x(t)=11$ casas/ano para 13 casas/ano, incorrerá num custo superior a $\$ 8.000,00$, para $T=45$ dias (fixo) e $V$ fixado em 13 unidades.

Proposição 3:

$$
\frac{\partial C}{\partial V}>0 \quad x=x_{0} ; T=T_{0}
$$

O custo aumenta com o volume de produção para $x(t)$ e $T$ dados, o período de colocação à disposição, $m$, sendo aumentado, por conseguinte. No caso presente, se $x(t)=11$ casas/ano, e se $T$ permanecer fixo em 45 dias, a passagem de $V=11$ unidades/ ano para 13 unidades/ano, acarretará, obviamente, uma elevação de custo.

Proposição 4:

$$
\frac{\frac{\partial C}{V}}{\partial V}<0 \quad T=T_{0}
$$

O custo médio diminui com o acréscimo do volume de produção $V$. Trata-se simplesmente do fenômeno de economia de escala, o qual pode ser obtido tanto pelo efeito obtido através da chamada "curva da experiência", quanto pela aprendizagem que os recursos humanos vão adquirindo ao longo do tempo. 


\section{Conclusão}

Em linhas gerais, admite-se que a gestão de projetos está principalmente direcionada à solução de problemas produtivos concernentes a situações inovadoras, isto é, encontradas em organizações cujas tarefas possuem elevado conteúdo de inovação.

Ademais, de acordo com Casarotto Filho (2002), a administração de projetos pode consideravelmente auxiliar as organizações que buscam posicionar-se melhor no mercado, proporcionando maior impulso às vantagens competitivas que ao longo do tempo a empresa deverá desenvolver: a saber, custo, qualidade, velocidade (tempo), confiabilidade, flexibilidade e inovações, em ordem crescente de importância quanto ao aspecto da dificuldade de gestão de sistema complexos (LIMA, 2002).

Constata-se ainda a relevância do trade-off entre custos de compressão de projetos e prazo de entrega. Assim, as técnicas de programação linear exibidas neste artigo se constituem numa ferramenta essencial para a obtenção de referências extremamente úteis às atividades de planejamento, programação e controle de sistemas produtivos. No entanto, o custo total estimado para a finalização do projeto dificilmente apresentará o mesmo valor do custo real incorrido.

A consideração das leis de custos poderá contribuir para a superação dessa lacuna, mediante a análise de outras variáveis importantes, tais como o volume global de produção $(V)$ e a data em que a primeira unidade do produto estará disponível para venda $(T)$.

Em se tratando do projeto de construção de um produto único, como é o caso da produção de hidrelétricas, por exemplo, essas variáveis não apresentam grande importância; todavia, para o projeto da casa adotado neste artigo, as variáveis $V$ e $T$ podem se tornar muito interessantes.

Assim, o custo médio do programa de produção diminui quando o volume global de produção $(V)$ e a taxa de produção $x(t)$ aumentam, ficando $T$ e $m$ fixos. Da mesma forma, o custo do programa de produção diminui quando a variável $T$ aumenta, reduzindo então a taxa de produção $x(t)$, ao permanecerem constantes as demais variáveis. Isto ocorre como corolário da proposição 2 . Com efeito, quanto maior for o valor de $T$, menor será o valor da taxa à qual os inputs são comprados, menor, então, são seus preços, porque são menores os custos de seus vendedores quando a proposição 2 lhes é aplicada, e menor é o custo do programa de produção.

Finalizando, os sistemas irregulares e de produção são não-lineares, na medida em que várias relações entre seus elementos são do tipo não-linear, tais como as funções de custos apresentadas neste trabalho. Em conseqüência, a intensidade do efeito nem sempre é proporcional à magnitude das causas e existe um amplo espectro de comportamentos possíveis. Nesse sentido, geralmente, os projetos na construção civil apresentam relações não-lineares, comumente observadas em sistemas complexos, sendo a formulação de um modelo de Dinâmica de Sistemas 
(DS) a representação mais apropriada de tal tipo de relação. A DS pode ser definida como uma metodologia para análise de sistemas dinâmicos complexos, apresentando múltiplas variáveis interdependentes, laços de realimentações e atrasos entre elas (SANTOS,2006).

$\mathrm{Na}$ maioria das vezes, os projetos tratam tanto informações qualitativas quanto quantitativas e a DS permite a modelagem de sistemas abordando ambas as variáveis, representando e quantificando as relações qualitativas existentes. Em suma, a metodologia tradicional de gerenciamento de projetos - composta por método do caminho crítico, gráfico de Gantt e método da força bruta, entre outros-possibilita apenas a manipulação de variáveis quantitativas, que embora apresentem relevância considerável, podem acarretar sérias limitações, uma vez que as ferramentas supracitadas consideram os projetos como sendo estáticos. Assim, a metodologia tradicional de gestão de projetos pode ser complementada pela DS, que por falta de espaço não será explicitada aqui.

\begin{abstract}
This paper looks for to add contributions of the Industrial Economics to the techniques used in the domain of the Projects Management. First, it is presented the technique of the brute force directed to the building and improved by the use of the MSProject. Second, models of least possible time for a project's completion and of programming the project's crashing that would implicate the least additional cost are developed. Third, laws of costs are established that allow inferring that the cost of a project does not depend only on the production tax. Concluding, the importance of the Management of Project with respect to the search of dynamic and innovative solutions to the more important productive problems of this age of the knowledge is pointed out.
\end{abstract}

Keywords: projects crashing, building, irregular systems of production.

\title{
Referências
}

ALCHIAN, A. Economic Forces at Work. Indianapolis: Liberty Press, 1977.

CASAROtTo Filho, N., FÁVERo, JOSÉ S.,\& CASTRO, JOÃo, E.E. Gerência de Projetos. Editora Decisoft LTDA, Florianópolis, 1992.

CASSAROTto FILHO, N. Projeto de Negócio: Estratégias e Estudos de Viabilidade. São Paulo: Editora Atlas, 2002.

FIGUEIREDO, FRANCISCO C. de, \& FIGUEIREDO, HELIO. MS Project 98 utilização na Gerência de Projetos. Rio de Janeiro: Editora Infobook, 1999.

GAFFARD, J. L. Economie Industrielle et de Línnovation. Paris: Editora Dalloz, 1990.

LIMA, MÁRCIO B. DA F. Groupware, uso das Tecnologias da Informação e Organização do Trabalho: Contribuições à Economia da Inovação. Tese de Doutorado. Florianópolis: UFSC, 2002.

MATTA, NADIM F. \& ASHKENAS, RONALD N. Gestão e Implementação de Projetos. Rio de Janeiro: Editora Campus, 2005.

MENEZES, LUÍS C. de M. Gestão de Projetos. São Paulo: Editora Atlas, 2003. 
RAGSDALE, CLIFF T. Spreadsheet Modeling and Decision Analysis - A practical introduction to the management science. 3. ed. New York: Southwestern Colleg Publishing, 2001.

SANDRONI, PAUlO. Novísssimo Dicionário de Economia. 3. ed. São Paulo: Editora Best Seller, 1999.

SANTOS, Alchen Montenegro dos. Aplicação de um modelo de simulação para o gerenciamento de Projetos: um estudo de caso utilizando a Dinâmica de Sistemas. Dissertação de mestrado apresentada ao Departamento de Engenharia Naval e Oceania da Escola Politécnica da Universidade de São Paulo, 2006.

VARGAS, RICARDO V. Microsoft Office Project 2003 - conhecendo a principal ferramenta de gerenciamento de projetos de mercado. Rio de Janeiro: Brasport, 2004.

WRITH, ALMIR. Planejando, replanejando e controlando com MS Project 2000. 2. ed. Rio de Janeiro: Editora Book Express, 2002.

\section{Nome Completo: MARCIO BOTELHO DA FONSECA LIMA}

Filiação institucional: Universidade Federal da Paraíba

Departamento: Departamento de Engenharia de Produção

Função ou cargo ocupado: Professor Assossiado II

Endereço: Rua Dr. Alcebíades Silva, 121/202, Cabo Branco, João Pessoa/Pb. Cep: 58.045-340

Cep: 58.051-970

Telefone: (83) 32270381

e-mail: tismalu@uol.com.br

Nome Completo: LUIZ BUENO DASILVA

Filiação institucional: Universidade Federal da Paraíba

Departamento: Departamento de Engenharia de Produção

Função ou cargo ocupado: Professor Adjunto IV

Endereço: Av. Acre, 130, apto 201, Ed. Rosa Leal, Bairro dos Estados, João Pessoa, PB, CEP número 58030-230

Telefone: 8332444166

e-mail: bueno@ct.ufpb.br

\section{Nome Completo: GLAUCO S. R. GOMES}

Filiação institucional: Universidade Federal da Paraíba

Departamento: Departamento de Engenharia de Produção

Função ou cargo ocupado: Graduando em Engenharia de Produção 
Endereço: Campus I, Bloco G, LabMEQA, Departamento de Engenharia de Produção, CT/UFPB, Cep número 58051-970

Telefone: 8332167685

e-mail: gomesjpa@terra.com.br 